\title{
Erratum: Quantum Criticality in Resonant Andreev Conduction [Phys. Rev. Lett. 119, 116802 (2017)]
}

\author{
M. Pustilnik, B. van Heck, R. M. Lutchyn, and L. I. Glazman
}

(Received 14 December 2017; published 10 January 2018)

DOI: 10.1103/PhysRevLett.120.029901

Equation (14) should read

$$
\frac{G}{G_{0}}=1-a \frac{T}{T_{K}}, \quad a \sim 1 ;
$$

i.e., the leading finite-temperature correction is linear in $T$. The error in Eq. (14) came from us disregarding the effect of the least-irrelevant perturbation of the Emery-Kivelson Hamiltonian. It is the same perturbation which produces the correct low-temperature asymptote of the specific heat in the two-channel Kondo model and which was identified in Ref. [1]. A standard perturbative calculation of the conductance then yields [2,3] a linear-in- $T$ dependence of $G$. Details can be found in Sec. 4B of Ref. [3].

The very same result can be obtained in the framework of resonant tunneling of Luttinger liquid characterized by parameter $K=1 / 2$. The least-irrelevant perturbation to the zero-energy fixed point is different from the $4 k_{F}$ scattering which we quoted after Eq. (14). Instead, the leading perturbation, identified in Ref. [4], has the form of properly symmetrized product of the local density $[\hat{n}(0)]$ and one-particle backscattering $\left(\psi_{L}^{\dagger} \psi_{R}\right)$ operators. The two perturbations yield, respectively, the corrections proportional to $T^{2(4 K-1)}$ and $T^{2 K}$ to the zero-temperature conductance. At $K=1 / 2$, the latter one yields the dominant, linear-in- $T$ term; its counterpart in the "strong tunneling" limit was obtained in Ref. [5].

The remaining results of our Letter remain unaffected.

We thank Harold Baranger, Christophe Mora, and Eran Sela for pointing out the error and helping us understand its origin.

[1] A. M. Sengupta and A. A. Georeges, Phys. Rev. B, 49, 10020 (1994).

[2] C. Mora (private communication).

[3] H. Zheng, S. Florens, and H. U. Baranger, Phys. Rev. B 89, 235135 (2014).

[4] S. Eggert and I. Affleck, Phys. Rev. B 46, 10866 (1992).

[5] A. Furusaki and K. A. Matveev, Phys. Rev. B 52, 16676 (1995); see Eq. (A9) with $N=1 / 2$ and $\left|r_{L}\right|=\left|r_{R}\right|$ therein. 